\title{
The mutational and phenotypic spectrum of TUBA1A-associated tubulinopathy
}

\author{
Moritz Hebebrand ${ }^{1}$, Ulrike Hüffmeier ${ }^{1}$, Regina Trollmann², Ute Hehr ${ }^{3}$, Steffen Uebe ${ }^{1}$, Arif B. Ekici', Cornelia Kraus ${ }^{1}$, \\ Mandy Krumbiegel ${ }^{1}$, André Reis ${ }^{1}$, Christian T. Thiel ${ }^{{ }^{* \dagger}}$ (ID and Bernt Popp ${ }^{1+}$
}

\begin{abstract}
Background: The TUBATA-associated tubulinopathy is clinically heterogeneous with brain malformations, microcephaly, developmental delay and epilepsy being the main clinical features. It is an autosomal dominant disorder mostly caused by de novo variants in TUBATA.

Results: In three individuals with developmental delay we identified heterozygous de novo missense variants in TUBA1A using exome sequencing. While the c.1307G > A, p.(Gly436Asp) variant was novel, the two variants c. 518C > T, p.(Pro173Leu) and c.641G > A, p.(Arg214His) were previously described. We compared the variable phenotype observed in these individuals with a carefully conducted review of the current literature and identified 166 individuals, 146 born and 20 fetuses with a TUBA1A variant. In 107 cases with available clinical information we standardized the reported phenotypes according to the Human Phenotype Ontology. The most commonly reported features were developmental delay (98\%), anomalies of the corpus callosum (96\%), microcephaly (76\%) and lissencephaly (agyria-pachygyria) (70\%), although reporting was incomplete in the different studies. We identified a total of 121 specific variants, including 15 recurrent ones. Missense variants cluster in the C-terminal region around the most commonly affected amino acid position Arg402 (13.3\%). In a three-dimensional protein model, $38.6 \%$ of all disease-causing variants including those in the C-terminal region are predicted to affect the binding of microtubule-associated proteins or motor proteins. Genotype-phenotype analysis for recurrent variants showed an overrepresentation of certain clinical features. However, individuals with these variants are often reported in the same publication.
\end{abstract}

Conclusions: With 166 individuals, we present the most comprehensive phenotypic and genotypic standardized synopsis for clinical interpretation of TUBATA variants. Despite this considerable number, a detailed genotypephenotype characterization is limited by large inter-study variability in reporting.

Keywords: TUBATA, Tubulin, Tubulinopathy, Lissencephaly, Brain malformation, Microcephaly, Developmental delay, Human phenotype ontology

\section{Introduction}

The superfamily of tubulin genes is composed of alpha-, beta-, gamma-, delta- and epsilon families. The alpha and beta families, consisting of at least 15 alpha and 21 beta-tubulin genes, respectively [1], encode tubulin proteins which form heterodimers as fundamental components of microtubules [2]. Along with microtubule associated proteins (MAPs) and motor proteins on the

\footnotetext{
* Correspondence: Christian.Thiel@uk-erlangen.de

${ }^{\dagger}$ Christian T. Thiel and Bernt Popp contributed equally to this work ${ }^{1}$ Institute of Human Genetics, Friedrich-Alexander-Universität

Erlangen-Nürnberg (FAU), Schwabachanlage 10, 91054 Erlangen, Germany Full list of author information is available at the end of the article
}

external surface, tubulin proteins participate in substantial cellular processes of intracellular transport, cell division and neuronal migration $[3,4]$.

In recent years, an increasing number of tubulin genes were linked to a clinically heterogeneous group of disorders, the "tubulinopathies" (TUBA1A, MIM\#602529; TUBA8, MIM\#605742; TUBB2A, MIM\#615101; TUBB2B, MIM\#612850; TUBB3, MIM\#602661; TUBB, MIM\#19 1130; TUBG1, MIM\#191135) [5-11]. Tubulinopathies are characterized by a broad spectrum of cortical and subcortical malformations and a variety of clinical features. Major cortical anomalies include lissencephaly (agyria-pachygyria), polymicrogyria or polymicrogyria-like cortical

(c) The Author(s). 2019 Open Access This article is distributed under the terms of the Creative Commons Attribution 4.0 International License (http://creativecommons.org/licenses/by/4.0/), which permits unrestricted use, distribution, and reproduction in any medium, provided you give appropriate credit to the original author(s) and the source, provide a link to the Creative Commons license, and indicate if changes were made. The Creative Commons Public Domain Dedication waiver (http://creativecommons.org/publicdomain/zero/1.0/) applies to the data made available in this article, unless otherwise stated. 
dysplasia and cortical gyral simplification. Subcortical anomalies affect the corpus callosum, the cerebellar vermis, the brainstem, the basal ganglia and the cerebellum. Further clinical features are microcephaly, global developmental delay and epilepsy $[12,13]$. To date, TUBA1A represents the main tubulinopathy gene and accounts for 4$5 \%$ of all lissencephaly cases $[14,15]$.

Using exome analysis in three unrelated individuals with severe developmental delay we identified three heterozygous de novo missense variants in the TUBA1A gene. We extensively reviewed and systematically reanalyzed available public data to provide a standardized synopsis of described variants together with reported neuroradiological and clinical features of TUBA1A-associated tubulinopathy. We used this comprehensive information to perform a detailed analysis of the genotypic and phenotypic spectrum highlighting a possible genotype-phenotype relationship and probable bias in reporting.

\section{Materials and methods Clinical reports of 3 novel cases}

For the purpose of this study the clinical course of three individuals, who presented between 1999 and 2016 at our Center of Developmental Neurology and Social Pediatrics for investigation of the etiology of developmental delay, was retrospectively summarized after pathogenic missense variants in TUBA1A had been identified. In summary, we present novel clinical data for two boys aged 13 years 7 months (individual i084n) and 11 years 6 months (individual i085n) and a 9 years 3 months old girl (individual i086n) with global developmental delay and neuroradiological abnormalities due to TUBA1A-associated tubulinopathy. The identification of the TUBA1A variant in the girl was part of a previous publication without detailed clinical description (reported as ID S_006) [16]. Narrative case reports with representative MRI planes for all three individuals and facial phenotype pictures for i086n (Additional file 1: Figure S1-S3) are provided in the Supplementary notes.

\section{Exome sequencing}

Informed written consent was obtained for all participants. The study was approved by the Ethical Committee of the Medical Faculty of the Friedrich-Alexander-Universität Erlangen-Nürnberg. DNA from peripheral blood lymphocytes was extracted using standard methods. Exome sequencing was performed after SureSelect v5 (i085n, i086n) and v6 (i084n) targeted capturing on HiSeq 2500 for i084n and i085n (Trio analysis [17]) and i086n (Exome Pool-Seq [16]). After mapping of sequence reads to the GRCh37/ hg19 reference genome and variant calling using standard methods for the trio analysis [17] or as described by Popp et al. for the exome Pool-Seq [16], variants in coding regions including splice sites were selected based on population frequency (gnomAD) and computational prediction scores, e.g. CADD score [18]. Variants were confirmed, and segregation tested by Sanger sequencing.

\section{Review of reported TUBA1A cases from literature and databases}

We identified 112 articles, published between 01/2007 and 06/2018, from PubMed applying the search term "TUBA1A". Of these, 28 provided clinical reports and were thus included in this study. All available clinical data was standardized in accordance to terms of the $\mathrm{Hu}$ man Phenotype Ontology (HPO) [19]. In contrast to a previously established classification combining cortical and subcortical features like "classic lissencephaly", "lissencephaly with cerebellar hypoplasia", "lissencephaly with agenesis of the corpus callosum" and "centrally predominant pachygyria" $[12,20]$, we analyzed the features independently. If only the classification was mentioned, we used the independent underlying features where HPO terms were available (e.g. "microlissencephaly": microcephaly HP:0000252 + agyria HP:0031882). Nevertheless, we kept composite terms typically used together in the literature such as "agyria-pachygyria" (HP:0031882, HP:0001302) if they affected the same brain structure. Data assessment comprised 11 neuroradiological features, including anomalies of cortical gyration, corpus callosum, brainstem, basal ganglia, internal capsule, cerebellum, cerebellar vermis, hippocampus, ventricular dilatation, 4th ventricle dilatation, grey matter heterotopia, and other radiological findings. Clinical features included congenital microcephaly, microcephaly, developmental delay, epilepsy, neuro-ophthalmological findings including strabismus and nystagmus, other neurological symptoms including spasticity and muscular hypotonia, and additional features (HPO terms shown in Tables 1 and 2, Additional file 2).

We further included available likely pathogenic or pathogenic variants from ClinVar [21], denovo-db [22] and DECIPHER [23]. As phenotype information was insufficient in most of these database cases, only variant information was included.

All variants were harmonized to the NM_006009.3 transcript of the GRCh37/hg19 human reference genome based on Human Genome Variation Society (HGVS) recommendations using the Mutalyzer [24] web services. To ensure consistency in the clinical interpretation we independently applied the American College of Medical Genetics and Genomics (ACMG) criteria [25] to all variants with the WGLAB InterVar-tool [26].

Protein structure analysis of the tubulin alpha-1A variants Using R and ggplot2 [27] we analyzed spatial distribution of all variants in the linear gene model to provide an insight into the variant distribution. Utilizing Pymol 
Table 1 Neuroradiological features of TUBATA-associated tubulinopathy

\begin{tabular}{|c|c|c|c|}
\hline Clinical information & Born n (\%) & Fetuses n (\%) & Total n (\%) \\
\hline Number of reported cases & $87(100.0)$ & $20(100.0)$ & $107(100.0)$ \\
\hline Sex & $34 \mathrm{f} / 38 \mathrm{~m} / 15 \mathrm{~ns}$ & $7 f / 12 \mathrm{~m} / 1 \mathrm{~ns}$ & $41 \mathrm{f} / 50 \mathrm{~m} / 16 \mathrm{~ns}$ \\
\hline Abnormality of the Corpus Callosum (HP:0001273) & $82 / 86(95.3)$ & $20 / 20(100.0)$ & 102/106 (96.2) \\
\hline Agenesis (HP:0001274) & 16/86 (18.6) & $16 / 20(80.0)$ & $32 / 106(30.2)$ \\
\hline Partial agenesis (HP:0001338) & $14 / 86(16.3)$ & $1 / 20(5.0)$ & 15/106 (14.2) \\
\hline Dysplastic (HP:0006989) & $14 / 86(16.3)$ & $3 / 20(15.0)$ & $17 / 106(16.0)$ \\
\hline Hypoplasia (HP:0002079) & $33 / 86(38.4)$ & $0 / 20(0.0)$ & $33 / 106(31.1)$ \\
\hline Partial agenesis, hypoplastic (HP:0001338, HP:0002079) & $5 / 86(5.8)$ & $0 / 20(0.0)$ & $5 / 106(4.7)$ \\
\hline Normal & $4 / 86(4.7)$ & $0 / 20(0.0)$ & $4 / 106(4.7)$ \\
\hline No information available & $1 / 87(1.2)$ & $0 / 20(0.0)$ & $1 / 107(0.9)$ \\
\hline Abnormal cortical gyration (HP:0002536) & 76/77 (98.7) & 19/19 (100.0) & 95/96 (99.0) \\
\hline Lissencephaly (HP:0006818) & $50 / 77(64.9)$ & $17 / 19(89.5)$ & $67 / 96(70.0)$ \\
\hline Agyria (HP:0031882) & $12 / 77(15.6)$ & 15/19 (78.9) & $27 / 96(28.1)$ \\
\hline Agyria-pachygyria (HP:0031882, HP:0001302) & $15 / 77(19.5)$ & $1 / 19(5.3)$ & $16 / 96(16.7)$ \\
\hline Pachygyria (HP:0001302) & 23/77 (29.9) & $1 / 19(5.3)$ & 24/96 (25.0) \\
\hline Polymicrogyria (HP:0002126) & 16/77 (20.8) & $2 / 19(10.5)$ & 18/96 (18.8) \\
\hline Perisylvian-polymicrogyria (HP:0012650) & 10/77 (13.0) & $0 / 20(0.0)$ & 10/96 (10.4) \\
\hline Cortical gyral simplification (HP:0009879) & $5 / 77(6.5)$ & $0 / 20(0.0)$ & $5 / 96(5.2)$ \\
\hline Unspecific & $5 / 77(6.5)$ & 0/20 (0.0) & $5 / 96(5.2)$ \\
\hline Normal & $1 / 77(1.3)$ & $0 / 20(0.0)$ & $1 / 96(1.0)$ \\
\hline No information available & 10/87 (11.5) & $1 / 20(5.0)$ & $11 / 107(10.3)$ \\
\hline Abnormality of the cerebellar vermis (HP:0002334) & 60/64 (93.8) & 18/18 (100.0) & 78/83 (94.0) \\
\hline Hypoplasia (HP:0001320) & $44 / 64(68.8)$ & $12 / 18(66.7)$ & $56 / 83(67.5)$ \\
\hline Dysgenesis (HP:0002195) & $16 / 64(25.0)$ & $6 / 18(33.3)$ & $22 / 83(26.5)$ \\
\hline Normal & $5 / 64(7.8)$ & $0 / 20(0.0)$ & $5 / 83(6.0)$ \\
\hline No information available & 22/87 (25.3) & $2 / 20(10.0)$ & 24/107 (22.4) \\
\hline Abnormality of the basal ganglia (HP:0002134) & $50 / 50(100.0)$ & $8 / 9(88.9)$ & $58 / 59(98.3)$ \\
\hline Dysgenesis (HP:0025102) & $50 / 50(100.0)$ & 8/9 (88.9) & $58 / 59(98.3)$ \\
\hline Normal & $0 / 50(0.0)$ & $1 / 9(11.1)$ & $1 / 59(1.7)$ \\
\hline No information available & $37 / 87(42.5)$ & $11 / 20(55.0)$ & 48/107 (44.9) \\
\hline Abnormality of the brainstem (HP:0002363) & $39 / 47(83.0)$ & 18/18 (100.0) & $57 / 65(87.7)$ \\
\hline Hypoplasia (HP:0002365) & $24 / 47(51.1)$ & $8 / 18(44.4)$ & $32 / 65(49.2)$ \\
\hline Pons hypoplasia (HP:0012110) & $6 / 47(12.8)$ & 10/18 (55.6) & $16 / 65(24.6)$ \\
\hline Dysplasia (HP:0002508) & $9 / 47(19.1)$ & $0 / 20(0.0)$ & 9/65 (13.8) \\
\hline Normal & $8 / 47(17.0)$ & $0 / 20(0.0)$ & $8 / 65(12.3)$ \\
\hline No information available & $40 / 87(46.0)$ & $2 / 20(10.0)$ & $42 / 107(39.3)$ \\
\hline Ventricular dilatation (HP:0002119) & $43 / 43(100.0)$ & $6 / 6(100.0)$ & $49 / 49(100.0)$ \\
\hline Fourth ventricle dilatation (HP:0002198) & 19/43 (44.2) & $1 / 6(16.7)$ & 20/49 (40.8) \\
\hline No information available & $44 / 87(50.6)$ & $14 / 20(70.0)$ & $58 / 107(54.2)$ \\
\hline Abnormality of the cerebellum (HP:0001317) & $22 / 32(68.8)$ & $16 / 17(94.1)$ & 38/49 (77.6) \\
\hline Dysplasia (HP:0007033) & $4 / 32(12.5)$ & $6 / 17(35.3)$ & 10/49 (20.4) \\
\hline Hypoplasia (HP:0001321) & $16 / 32(50.0)$ & 10/17 (58.8) & $26 / 49(53.1)$ \\
\hline Agenesis (HP:0012642) & $1 / 32(3.1)$ & $0 / 20(0.0)$ & $1 / 49(2.0)$ \\
\hline Normal & 10/32 (31.3) & $1 / 17(5.9)$ & $11 / 49(22.4)$ \\
\hline
\end{tabular}


Table 1 Neuroradiological features of TUBA1A-associated tubulinopathy (Continued)

\begin{tabular}{|c|c|c|c|}
\hline Clinical information & Born n (\%) & Fetuses n (\%) & Total n (\%) \\
\hline No information available & $55 / 87(63.2)$ & $3 / 20(15.0)$ & $58 / 107(54.2)$ \\
\hline Abnormal morphology hippocampus (HP:0025100) & $24 / 29(82.8)$ & $5 / 8(62.5)$ & 30/38 (78.9) \\
\hline Hypoplasia (HP:0025517) & $6 / 29(20.7)$ & $3 / 8(37.5)$ & 9/38 (23.7) \\
\hline Dysgenesis (HP:0025101) & $18 / 29(62.1)$ & $2 / 8(25.0)$ & 20/38 (52.6) \\
\hline Normal & $5 / 29(17.2)$ & $3 / 8(37.5)$ & $8 / 38(21.1)$ \\
\hline No information available & $58 / 87(66.7)$ & $12 / 20(60.0)$ & $69 / 107(64.5)$ \\
\hline Abnormality of the internal capsule (HP:0012502) & $24 / 25(96.0)$ & $1 / 19(100.0)$ & $25 / 26(96.2)$ \\
\hline Anterior limb thinned or absent & $13 / 25(52.0)$ & $0 / 20(0.0)$ & $13 / 26(50.0)$ \\
\hline Normal & $1 / 25(4.0)$ & $0 / 20(0.0)$ & $1 / 26(3.8)$ \\
\hline No information available & $62 / 87(71.3)$ & 19/20 (95.0) & $81 / 107(75.7)$ \\
\hline Grey matter heterotopia (HP:0002281) & $11 / 13(84.6)$ & 14/15 (93.3) & $25 / 28(89.3)$ \\
\hline Olivary & $5 / 13(38.5)$ & $6 / 15(40.0)$ & $11 / 28(39.3)$ \\
\hline Absent & 2/13 (15.4) & $1 / 15(6.7)$ & $3 / 28(10.7)$ \\
\hline No information available & $74 / 87(85.1)$ & $5 / 20(25.0)$ & 79/107 (73.8) \\
\hline Other radiological features & $12 / 12(100)$ & 8/8 (100.0) & $20 / 20(100.0)$ \\
\hline Abnormal morphology of the olfactory bulb (HP:0040327) & $2 / 12(16.7)$ & $6 / 8(75.0)$ & $8 / 20(40.0)$ \\
\hline No information available & 75/87 (86.2) & $12 / 20(60.0)$ & $87 / 107(81.3)$ \\
\hline
\end{tabular}

F Female, $M$ Male, $N$ Number, N/A Not applicable, NS Not specified

(Version 1.8.6.0; Schrödinger, LLC) installed through Conda (Version 4.4.9 build 3.0.27 with Python 2.7.14; Anaconda Inc.) publicly available tertiary protein structure data of TUBA1A (PDB-ID: J5CO [28]) was used to classify variants in different groups of potential functional effects as suggested previously [29]. This classification is based on the interaction of the tubulin monomer with neighboring tubulin proteins within the polymer (heterodimer, protofilament, microtubule), with MAPs, or motor proteins. While functional evidence was present only for a minority of the variants $[5,30]$, most mutational effects are based on localization-dependent predictions. As a template we used 51 already classified TUBA1A variants [31] likely affecting the binding of microtubule associated proteins ("MAP binding") or motor proteins, the tubulin folding ("Tubulin folding"), heterodimer and microtubule stability ("Intradimer interaction" and "Longitudinal interaction") the formation of the hollow tubular structure of the microtubule ("Lateral interaction") [32, 33] or microtubule dynamics, protein folding and heterodimer stability ("GTP [Guanosintriphosphat] binding") [29, 32]. The specific detrimental effect of variants facing the luminal protein surface ("Lumen facing") is currently unknown.

\section{Computational analyses of TUBA1A missense variant spectrum}

We here analyzed the ability of six different computational classifiers (three ensemble scores: CADD, M-CAP, REVEL and the three commonly used scores Polyphen-2, SIFT,
MutationTaster) to discriminate pathogenic and neutral population variants by generating all possible missense variants for TUBA1A. First all single base exchanges were generated in the TUBA1A gene region of the GRCh37/ hg19 reference (chr12[hg19]:49578578-49,583,107) as variant call format (VCF) file. These were then annotated with computational scores and databases from dbNSFP [34] version 2.9.3 and variant frequencies from the gnomAD database [35] version 2.0.1 using SnpEff/SnpSift [36]. Missense variants affecting the NM_006009.3 transcript of TUBA1A, excluding variants, which were additionally annotated as potentially affecting splicing, were selected. R language [37] version 3.4.3 with RStudio IDE version 1.1.383 (RStudio, Inc.) with packages from the tidyverse/ggplot2 [27] collection were used for plotting and analysis of this variant data provided in the Additional file 2 . To analyze possible mutational hotspots, we generated density plots of pathogenic missense variant frequencies reported in the literature and missense variants reported in controls from gnom $A D$ with the "geom_density" function ("adjust" parameter set to $1 / 4$ ) in ggplot2. To analyze protein regions of higher conservation we plotted all missense variants sorted by amino-acid position with each respective computational score and fitted generalized additive models using the "geom_smooth" function in ggplot2 to produce a smoothed line. Additionally, variants and scores were plotted as scatter and violin plots and two-sided Wilcoxon signed-rank test from the ggsignif package was used to determine whether there was a statistically significant difference between four different 
Table 2 Clinical features of TUBA 1 A-associated tubulinopathy

\begin{tabular}{|c|c|c|c|}
\hline Clinical information & Born n (\%) & Fetuses n (\%) & Total n (\%) \\
\hline Number of reported cases & $87(100.0)$ & $20(100.0)$ & $107(100.0)$ \\
\hline Sex & $34 \mathrm{f} / 38 \mathrm{~m} / 15 \mathrm{~ns}$ & $7 f / 12 \mathrm{~m} / 1 \mathrm{~ns}$ & $41 \mathrm{f} / 50 \mathrm{~m} / 16 \mathrm{~ns}$ \\
\hline Microcephaly (HP:0000252) & $47 / 53(88.7)$ & $10 / 20(50.0)$ & $57 / 75(76.0)$ \\
\hline Normal & $8 / 53(15.1)$ & $10 / 20(50.0)$ & $18 / 75(24.0)$ \\
\hline No information available & $32 / 87(36.8)$ & $0 / 20(0.0)$ & 32/107 (29.9) \\
\hline Congenital microcephaly (HP:0011451) & $25 / 36(69.4)$ & $\mathrm{n} / \mathrm{a}$ & $25 / 36(69.4)$ \\
\hline Normal & $11 / 36(30.6)$ & $\mathrm{n} / \mathrm{a}$ & $11 / 36(30.6)$ \\
\hline No information available & $51 / 87(58.6)$ & $\mathrm{n} / \mathrm{a}$ & $51 / 87(58.6)$ \\
\hline Global developmental delay (HP:0001263) & $52 / 53(98.1)$ & $\mathrm{n} / \mathrm{a}$ & $52 / 53(98.1)$ \\
\hline Normal & $1 / 53(1.9)$ & $\mathrm{n} / \mathrm{a}$ & $1 / 87(1.1)$ \\
\hline No information available & $34 / 87(39.1)$ & $\mathrm{n} / \mathrm{a}$ & 34/87 (39.1) \\
\hline Other neurological symptoms & $39 / 40(97.5)$ & $\mathrm{n} / \mathrm{a}$ & $38 / 40(95.0)$ \\
\hline Spasticity (HP:0001257) & $19 / 40(47.5)$ & $n / a$ & $23 / 40(57.5)$ \\
\hline Muscular hypotonia (HP:0001252) & $10 / 40(25.0)$ & $\mathrm{n} / \mathrm{a}$ & $16 / 40(40.0)$ \\
\hline Spasticity and muscular hypotonia (HP:0001257, HP:0001252) & $6 / 40(15.0)$ & $\mathrm{n} / \mathrm{a}$ & $6 / 40(15.0)$ \\
\hline Other & $4 / 40(10.0)$ & $\mathrm{n} / \mathrm{a}$ & $4 / 40(10.0)$ \\
\hline Normal & $1 / 40(2.5)$ & $\mathrm{n} / \mathrm{a}$ & $1 / 40(2.5)$ \\
\hline No information available & $47 / 87(54.0)$ & $\mathrm{n} / \mathrm{a}$ & $47 / 87(54.0)$ \\
\hline Epilepsy (HP:0001250) & $37 / 51(72.5)$ & $\mathrm{n} / \mathrm{a}$ & $37 / 52(71.2)$ \\
\hline Generalized tonic-clonic seizures (HP:0002069) & 19/51 (37.3) & $\mathrm{n} / \mathrm{a}$ & 19/52 (36.5) \\
\hline Infantile spasms (HP:0012469) & $4 / 51(7.8)$ & $\mathrm{n} / \mathrm{a}$ & $4 / 52(7.7)$ \\
\hline Generalized tonic-clonic seizures and infantile spasms (HP:0002069, HP:0012469) & $5 / 51(9.8)$ & $\mathrm{n} / \mathrm{a}$ & $5 / 52(9.6)$ \\
\hline Focal seizures (HP:0007359) & $8 / 51(15.7)$ & $\mathrm{n} / \mathrm{a}$ & 9/52 (17.3) \\
\hline Absent & $15 / 51(29.4)$ & $\mathrm{n} / \mathrm{a}$ & $15 / 52(28.8)$ \\
\hline No information available & $35 / 87(40.2)$ & $\mathrm{n} / \mathrm{a}$ & $35 / 87(40.2)$ \\
\hline Neuroophtalmological features & $26 / 29(89.7)$ & $1 / 5(20.0)$ & 27/35 (77.1) \\
\hline Strabismus (HP:0000486) & $14 / 29(48.3)$ & $\mathrm{n} / \mathrm{a}$ & $14 / 35(40.0)$ \\
\hline Nystagmus (HP:0000639) & $3 / 29(10.3)$ & $\mathrm{n} / \mathrm{a}$ & $3 / 35(8.6)$ \\
\hline Strabismus and nystagmus (HP:0000486, HP:0000639) & $4 / 29(13.8)$ & $\mathrm{n} / \mathrm{a}$ & 4/35 (11.4) \\
\hline Optic nerve hypoplasia (HP:0008058) & $6 / 29(20.7)$ & $1 / 5(20.0)$ & $7 / 35(20.0)$ \\
\hline Absent & 4/29 (13.8) & $4 / 5(80.0)$ & $8 / 35(22.9)$ \\
\hline No information available & $57 / 87(65.5)$ & $15 / 20(75.0)$ & $72 / 107(67.3)$ \\
\hline Facial anomalies (HP:0000271) & $21 / 29(72.4)$ & $9 / 15(60.0)$ & $30 / 44(68.2)$ \\
\hline Micro-/retrognathia & $6 / 29(20.7)$ & $7 / 15(46.7)$ & $13 / 44(30.0)$ \\
\hline Absent & $8 / 29(27.6)$ & $6 / 15(40.0)$ & 14/44 (31.8) \\
\hline No information available & $58 / 87(66.7)$ & $5 / 20(25.0)$ & 63/107 (58.9) \\
\hline
\end{tabular}

$F$ Female, $M$ Male, $N$ Number, N/A Not applicable, NS Not specified

missense variant groups ("clinical review", "database", "gnomAD", "simulated”). "Clinical review" included variants from individuals with available phenotype information from our literature review and the three cases reported here, "database" included (likely) pathogenic variants from databases like ClinVar without clinical information, "gnomAD" included all variants present in healthy controls without neurodevelopmental disorders from the
gnomAD database, and "simulated" all other possible missense variants in TUBA1A.

\section{Analysis of genotype-phenotype relation}

We used the curated set of clinical information and corresponding harmonized variant information to analyze a possible genotype-phenotype relationship by comparing the radiological and clinical features with variant characteristics. 
We visualized and structured the acquired categorical data into a grid plot using ggplot2 and the tidyverse [27] package for hypothesis formation. Based on this presentation we used the vcd package [38] to analyze the relationship between variant characteristics and clinical data of the individuals by generating mosaic or association plots. As many values in the resulting contingency tables contained values below five, we estimated $p$-values using a two-sided Fisher's exact test with the "simulate.p.value" setting based on 2000 replicates in R. One-letter amino-acid nomenclature is used in the resulting plots because of space constrains.

\section{Results}

\section{Results of exome sequencing in $\mathbf{3}$ affected individuals}

We identified three heterozygous missense variants c. $518 \mathrm{C}>\mathrm{T}$, c.1307G > A, and c.641G > A in TUBA1A. Segregation analysis demonstrated that all variants were de novo. The missense variant c.518C > T, p.(Pro173Leu) identified in individual i084n and the missense variant c.641G > A, p.(Arg214His) in individual i086n, located in exon 4 of TUBA1A, were both previously reported either in an affected individual with autism spectrum disorder [39] (c.518C > T, p.(Pro173Leu)) or in several affected individuals with developmental delay and complex cerebral malformations $[20,40]$ (c.641G > A, p.(Arg214His)). The heterozygous missense variant c.1307G > A, p.(Gly436Asp) identified in individual i085n was absent in the unaffected parents (de novo, sample identity confirmed), not listed in gnomAD, located in a highly conserved domain and multiple lines of computational evidence predicted a deleterious effect. Thus, we classified all variants as pathogenic (class 5) in accordance with the ACMG criteria.

\section{Mutational spectrum and distribution of TUBA1A variants}

We retrieved a total of 61 distinct variants from 84 born individuals and 20 fetuses from 28 published articles in Pubmed and 59 further distinct variants from databases [5, 13, 15, 20, 30, 39-67]. Moreover we identified one novel variant c. $1307 \mathrm{G}>\mathrm{A}$, p.(Gly436Asp), not reported in databases or the literature, in one of the three herein described individuals. Of these 121 distinct variants 119 were missense and two led to a premature stop codon located at the C-terminal domain and are predicted to escape nonsense mediated decay. Common recurrent variants were c.1205G > A p.(Arg402His), c.1204C > T p.(Arg402Cys) and c.790C > T p.(Arg264Cys) reported 11, 8 and 10 times, respectively. The Arg402 residue is the most commonly (13.3\%) affected amino-acid position (Arg402His, Arg402Cys, Arg402Leu, Arg402Ser). After standardization to the ACMG criteria, 120 of the 121 distinct variants were classified as likely pathogenic or pathogenic (ACMG class 4 or 5) $(99.2 \%$ ) and one variant (c.1224C > A, p. $($ Tyr408*)) was classified as of unknown significance (VUS, ACMG class 3).
TUBA1A consists of the N-terminal, intermediate and C-terminal domains [68]. Annotation of variants on the linear gene model revealed that variants were distributed all over the TUBA1A gene with a statistically significant clustering around the Arg402 residue in exon 4 in the C-terminal domain. This cluster correlates with high computational prediction scores for missense variants (Fig. 1a, b and c; Additional file 1: Figure S4). Variants in the linear $\mathrm{C}$-terminal region predominantly affect the binding of MAPs or motor proteins. Strikingly, computational scores for the different missense variant groups ("clinical review", "database", "gnomAD", "simulated") mostly showed no significant difference (Fig. 1d; Additional file 1: Figure S4). After mapping of the amino acid residues on the 3D protein structure, we observed that most unique variants in "clinical review" $(n=121)$ are predicted to compromise tubulin folding (34.7\%) or possibly affecting the interaction with MAPs or motor proteins, such as kinesins and dyneins (24.8\%) (Fig. 2). A minority of variants is predicted to affect longitudinal (8.3\%), lateral (8.3\%) and intradimer (7.4\%) interactions, respectively. Finally, $14 \%$ of variants are lumen facing and only $2.5 \%$ likely affect GTP binding. Considering all assembled variants including the recurrent ones $(n=166)$, the majority (38.6\%) is predicted to impair the interaction of MAPs or motor proteins. Of these, 22 affect the Arg402 position. Variants identified in the three individuals i084n, i085n, i086n described here are predicted to affect tubulin folding (c.518C $>\mathrm{T}$, p.(Pro173Leu), MAP binding (c.1307G > A, p.(Gly436Asp) and intradimer interactions (c.641G > A, p.(Arg214His), respectively.

\section{Clinical spectrum of TUBA1A variants}

Based on available information, major neuroradiological features of TUBA1A-associated tubulinopathy include anomalies of the cortical gyration $(99.0 \%, 95 / 96)$, with lissencephaly [agyria-pachygyria (HP:0031882, HP:0001302)] and polymicrogyria reported in $70.0 \%(67 / 96)$ and $18.8 \%$ (18/96) respectively. Further anomalies affect the basal ganglia $(98.3 \%, 58 / 59)$, the corpus callosum $(96.2 \%, 102 /$ $106)$, the capsula interna $(96.2 \%, 25 / 26)$ and the cerebellar vermis $(94.0 \%, 78 / 83)$. Ventricular dilatation was reported in $100.0 \%(49 / 49)$ and anomalies of the hippocampus in $78.4 \%$ (30/38) (Table 1). Clinical features included global developmental delay $(98.1 \%, 52 / 53)$, microcephaly $(76.0 \%$, $57 / 75)$, epilepsy $(71.2 \%, 37 / 52)$ and spasticity $(62.5 \%, 25 /$ 40) (Table 2). Data missingness ranged from $0.9 \%$ (corpus callosum) to $75.7 \%$ (internal capsule) for neuroradiological features and from 29.9\% (microcephaly) to $67.3 \%$ (neuroophthalmological features) for clinical features. We provide a detailed summary of the currently described clinical features in born individuals and fetuses with details of data missingness in Tables 1 and 2. 


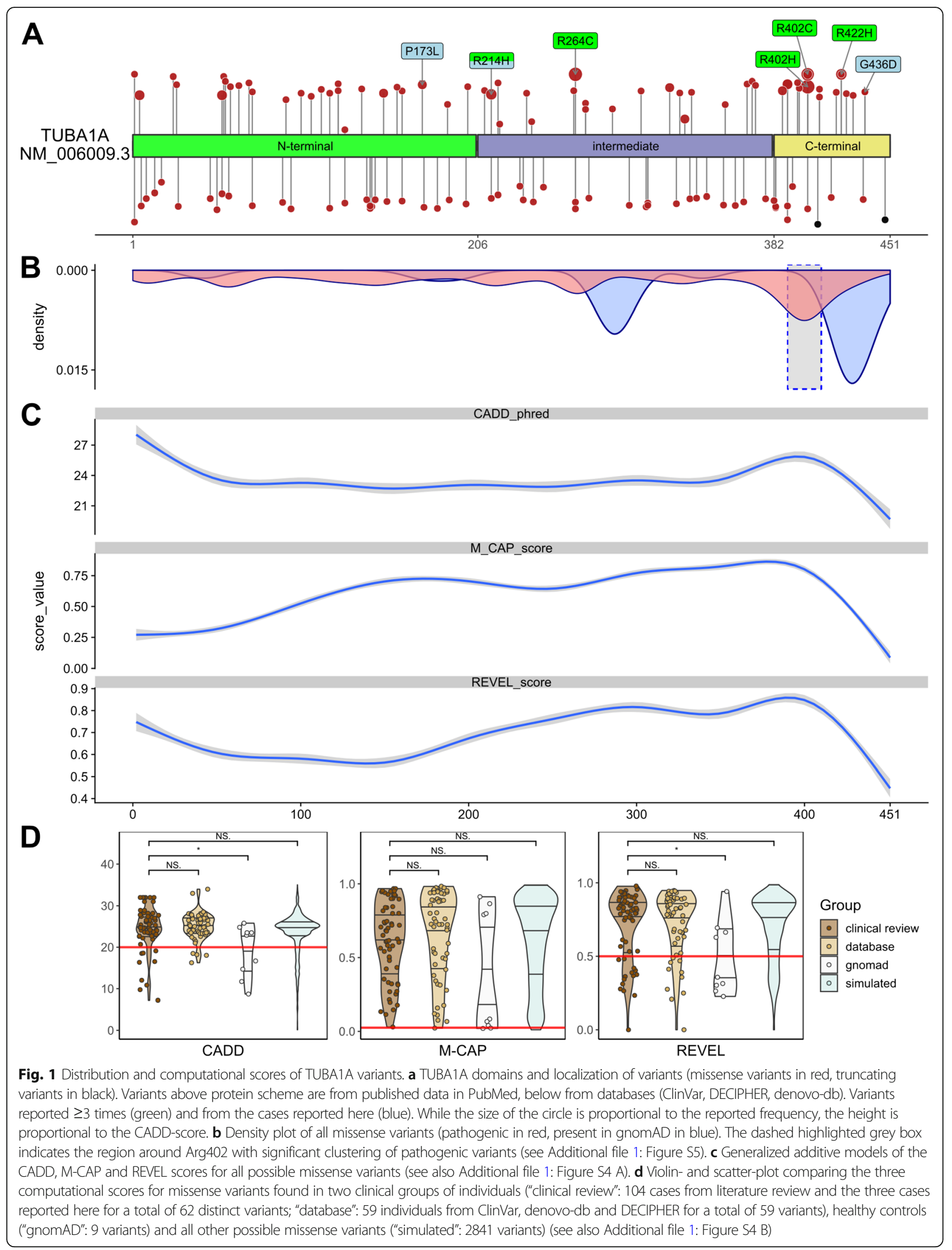




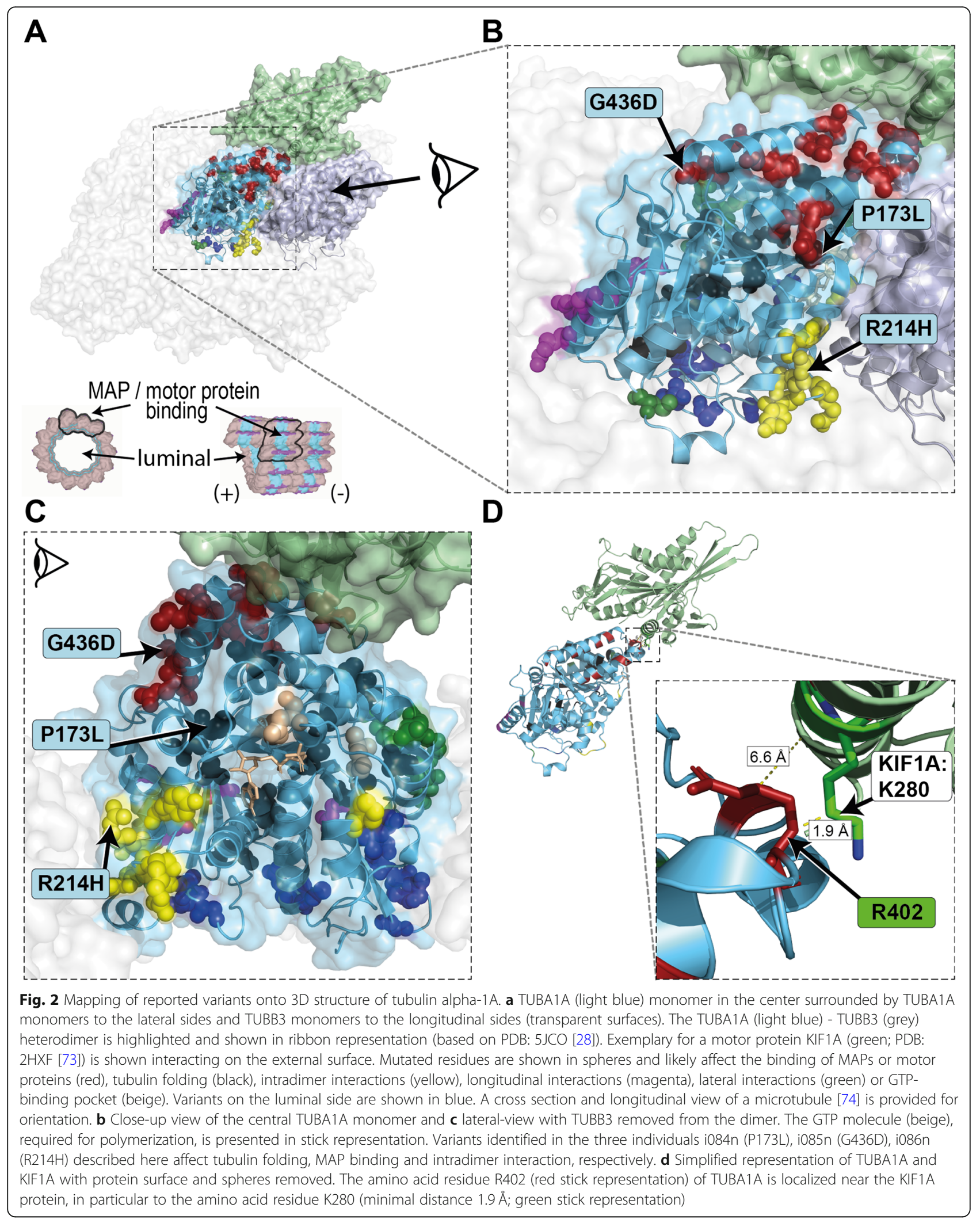




\section{Relation between genotype and phenotype}

We used the clinical information of the 104 individuals from the "clinical review" group and the herein described three patients (total $n=107$ ) to analyze a possible relationship between genotype and phenotype. Individuals with recurrent variants, mostly affecting MAP or motor protein binding, show a similar phenotype combination in the matrix plot (Fig. 3a; see also Additional file 1: Figure S7). Patients with the missense variant p.(Arg402Cys) are mostly described with a cortical-gyration pattern of agyria-pachgyria ("Ag-Pg"), dysplastic corpus callosum ("D"), a cerebellar vermis hypoplasia ("H") and have no information reported for the brainstem.

Because prenatally diagnosed fetal cases show a more severe phenotype than born individuals, we analyzed a possible contribution of variant characteristics to this observation. The missense variants reported in fetuses and in born individuals showed no significant difference in structural classification (Fig. 3b) and the computational scores did not significantly differ in these two groups (Additional file 1: Figure S6). In addition, the structural groups of missense variants were not overrepresented in females or males and the gender was also not associated with prenatal diagnosis (Fig. 3b).

The visual inspection of the matrix plot (Fig. 3a; Additional file 1: Figure S7) indicated that certain clinical features are enriched in individuals with recurrent variants. Indeed, explorative comparison between missense variants at recurrent and non-recurrent positions confirmed differences in reported clinical features of the individuals carrying these missense variants (Fig. 3c; Additional file 1: Figure S8). Despite our effort to collect all variants and clinical information described for TUBA1A-associated tubulinopathy, we did not obtain enough data to further analyze the phenotype differences for these variants.

Finally, we observed that individuals with the same variants and similar phenotypes were often reported together (e.g. Fig.3a "+" symbol for Arg402Cys reported 5 times in PMID:20466733 [30]). Regarding this observation, we found a significant difference in the use of clinical descriptions in publications describing multiple individuals. Kumar et al. (PMID:20466733 [30]) and Bahi-Buisson et al. (PMID:24860126 [13]) both describe four cases with the de novo missense variant c.1205G > A p.(Arg402His), but Bahi-Buisson et al. more often describes agyria ("Ag") as cortical gyration pattern. Romaniello et al. (PMID:28677066 [57]) describe perisylvian polymicrogyria ("PsMPG") as cortical gyration pattern for four (all with different missense variants) of their 14 reported individuals' variants while this term is only used for 6 other individuals in the entire "clinical review" group (Fig. 3d; Additional file 1: Figure S9).

\section{Discussion}

In this study, we identified three de novo missense variants in TUBA1A in three individuals with global developmental delay and brain malformations. Since the first identification of disease-causing variants in TUBA1A in 2007 in two affected individuals with cortical dysgenesis [5], at least 121 distinct heterozygous variants in a total of at least 166 patients including our 3 affected individuals are now described. Our efforts to systematically reanalyze published data enabled insights into the current state of information about TUBA1A-associated tubulinopathy.

Anomalies of the corpus callosum ranging from partial to complete agenesis or hypoplasia are with $96.2 \%$ (102/ 106) the predominantly reported feature of TUBA1Aassociated tubulinopathy. Cortical anomalies are the second leading clinical feature reported in 95/96 individuals (99.0\%) followed by dysgenesis of the basal ganglia in $58 / 59$ (98.3\%). Two of the herein described individuals (i084n, i085n) also presented these features. Individual i086n had complete agenesis corpus callosum and additionally manifested unilateral optic nerve hypoplasia, a feature linked to TUBA8-associated tubulinopathy [6] but also described in individuals with TUBB2B [69] and TUBB3 [9] variants and present in 7/35 (20.0\%) of individuals with TUBA1A-associated tubulinopathy.

Analysis of the type and localization of all possible 2969 missense variants from the simulation showed that the large majority of TUBA1A missense variants are predicted to be deleterious (CADD $\geq 20: 84.2 \%$, M-CAP $\geq 0,025$ : $98.0 \%$, REVEL $\geq 0,5: 78.8 \%$; Fig. $1 d$ ). This is in agreement with an ExAC Z-score [35] of 6.23, confirming that TUBA1A is extremely depleted of missense variants in the general population. This resulted in high computational prediction scores independent of causality. Thus, variants might be reported to be likely pathogenic or pathogenic (ACMG class 4 or 5) despite relatively low computational scores and variants found in healthy controls might have scores above the recommended respective thresholds (Fig. 1d). After analyzing the relation of three ensemble computational prediction scores and expected pathogenicity, we concluded that computational prediction scores are of limited utility for predicting pathogenicity in TUBA1A. We suggest that segregation with the disease in the family or de novo occurrence, two major criteria of the ACMG guidelines for variant interpretation, are more appropriate for variant classification.

Based on the observation of the mutational distribution we analyzed a possible relationship between genotype and phenotype. We observed clustering of disease-causing variants in the region around the amino acid residue Arg402 (Fig. 1a, b and c, Additional file 1: Figure S2). The residue Arg402 is located in the interaction site of various MAPs or motor proteins [30] 


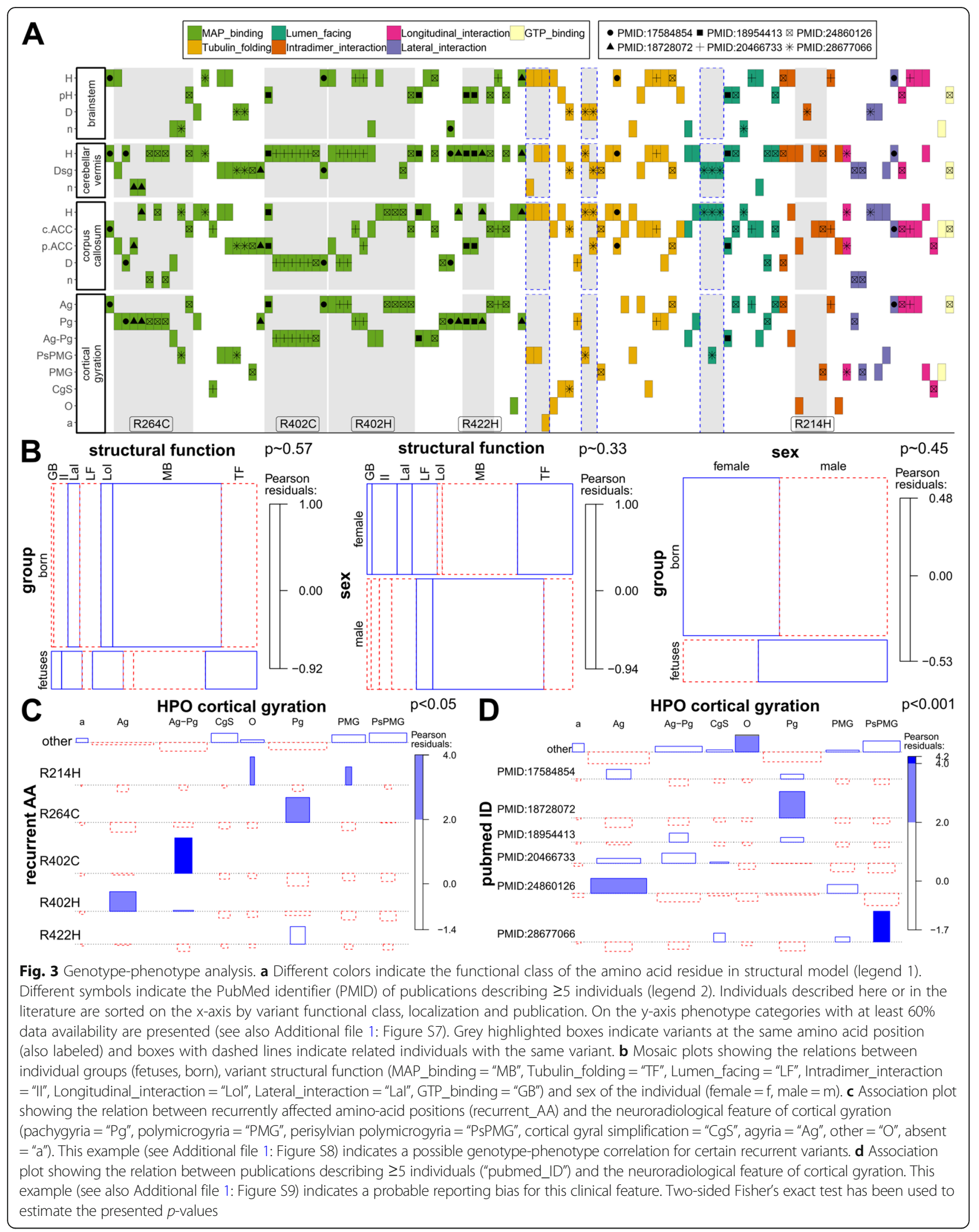


which are involved in different processes including the polymerization and stabilization of microtubules and intracellular vesicle transport [70]. Defects in some MAPs or motor proteins result in a similar clinical spectrum as observed for specific MAP-associated TUBA1A variants [30, 71]. Overall, variants of the Arg402 residue and other specific recurrent variants, which are predominantly MAP or motor protein interacting, were previously associated with overlapping neuro-radiological features [13, 30]. Indeed, we could show a non-uniform distribution for reported clinical features and the recurrent variants (Fig. 3c), indicating a possible genotype-phenotype relation. This observation might in part be attributed to detailed structured morphological categorization of brain anomalies used by different authors and individual preferences for certain terms. In addition, difficulties in the interpretation of the radiographic cortical and subcortical anomalies or technical differences in brain imaging could represent a possible confounder. Of note, recurrent variants with similar phenotype combinations were often reported by the same authors indicating a possible observational bias (Fig. 3d), thus limiting the interpretation of these genotype-phenotype relations. Another problem hindering a more detailed investigation is the high degree of missing data we recognized for several phenotypic categories. The directed acyclic graphs structure of HPO allows grouping of specialized terms into less specialized parent terms. Future development of algorithms comparing the phenotypic similarity between groups of individuals with the same or functionally similar pathogenic variants might alleviate some of these problems and allow further characterization of variant specific phenotypes. However, some of these endeavors could be hampered by the difficulty to distinguish between missing information and normal phenotype in published reports. This is especially problematic as HPO describes "phenotype abnormalities" but has no terms for normal phenotypes. We propose standardization in clinical reporting of rare disease cases based on expert recommendations with a minimal scheme covering disease specific phenotypes.

Even though TUBA1A-associated tubulinopathy is the most common tubulinopathy form, our results indicate that more clinical and mutational information is necessary to evaluate a potential genotype-phenotype correlation. This became apparent in fetuses, where we and others observed the most severe phenotypic spectrum compared to born cases $[13,20]$. This could not be explained by specific properties of the identified variants (Fig. 3b, Additional file 1: Figure S6). We therefore propose that additional variants in other genes or random developmental processes in cellular pathways in the respective individuals are underlying the phenotypic variability. Genome wide and functional studies might help to allow further characterization into specific clinical groups.

\section{Conclusion}

Our systematic reanalysis of published clinical data allowed an explorative investigation of a genotype-phenotype relationship. We found an enrichment of specific radiological features in recurrent variants; however, insufficient data availability, data variability and a possible observer bias were limiting factors for possible associations. A thoroughly conducted clinical examination and the standardized reporting of phenotype and genotype information in online databases, e.g. ClinVar [21] and LOVD [72] are fundamental for the systematic analysis of rare diseases such as TUBA1A-associated tubulinopathy.

\section{Additional files}

Additional file 1: Figure S1. Cranial MRI planes of individual i084n. Figure S2. Cranial MRI planes of individual i085n. Figure S3. Cranial MRI planes and clinical pictures of individual i086n. Figure S4. Additional computational scores for TUBA1A variants. Figure S5. Analysis of the variant cluster around amino acid position 400. Figure S6. Comparison of computational scores for TUBA1A variants identified in fetuses and born individuals. Figure S7. Matrix plot of all HPO phenotype categories. Figure S8. Association plots for recurrently affected amino-acid positions and all neuroradiological features. Figure S9. Association plots publications describing $\geq 5$ individuals and all neuroradiological features. Table S1. Barthel Index of Activities of Daily Living [3] of the 3 individuals with TUBA1A variants. (DOCX $2946 \mathrm{~kb}$ )

Additional file 2: Additional information, phenotypes and TUBA1A variants. (XLSX $574 \mathrm{~kb}$ )

\section{Abbreviations}

ACMG: American College of Medical Genetics and Genomics; CMA: Chromosomal microarray; GTP: Guanosintriphosphat; HGVS: Human Genome Variation Society; HPO: Human Phenotype Ontology; MAPs: Microtubule-associated proteins; MRI: Magnetic resonance imaging; OFC: Occipitofrontal circumference; VCF: Variant call format

\section{Acknowledgements \\ We thank all participants and their families for taking part in this study. We acknowledge the excellent technical support of Angelika Diem, and Heike Friebel-Stange. We also thank the Exome Aggregation Consortium and the groups that provided exome variant data for comparison. A full list of contributing groups can be found at http://exac.broadinstitute.org/about. This study utilized data generated by the DECIPHER community. A full list of centers that contributed to data generation is available online from http:// decipher.sanger.ac.uk and via email from decipher@sanger.ac.uk.}

\section{Funding}

This study was supported by grants from the German Research Foundation (DFG; grants TH 896/3-4), the Interdisciplinary Centre for Clinical Research (IZKF) of the Friedrich-Alexander-Universität Erlangen-Nürnberg (Project F4) and by the ELAN Fonds (14-08-06-1) of the Faculty of Medicine of the Friedrich-Alexander Universität Erlangen-Nürnberg (FAU) to $\mathrm{CT}$.

Availability of data and materials

All data generated or analysed during this study are included in this published article [and its Additional file 2]. All data is available from the corresponding author on request. 


\section{Web resources}

gnomAD browser: http://gnomad.broadinstitute.org/. Mutalyzer: https:// mutalyzer.nl/. winterVar: http://wintervar.wglab.org/

\section{Authors' contributions}

$\mathrm{CT}$ and $\mathrm{UH}$ provided all clinical findings and patient samples. MK conducted Array analyses. CK, SU, AE, CT, AR and BP analyzed and interpreted the sequencing data. BP created Figs. 1, 2 and 3 and the supplementary materials. $\mathrm{MH}$ performed the literature review and standardization to HPO terms. $\mathrm{MH}$ and $\mathrm{BP}$ created Fig. 2. BP, MH and $\mathrm{CT}$ wrote and edited the manuscript, $C K, M K, S U, A E, C T$, AR and $B P$ reviewed the draft manuscript. All authors read and approved the final manuscript.

\section{Ethics approval and consent to participate}

Informed written consent was obtained for all participants. The study was approved by the Ethical Committee of the Medical Faculty of the FriedrichAlexander-Universität Erlangen-Nürnberg (180_15BC).

\section{Consent for publication}

Informed written consent for publication was obtained for all participants.

\section{Competing interests}

The authors declare that they have no competing interests.

\section{Publisher's Note}

Springer Nature remains neutral with regard to jurisdictional claims in published maps and institutional affiliations.

\section{Author details}

'Institute of Human Genetics, Friedrich-Alexander-Universität Erlangen-Nürnberg (FAU), Schwabachanlage 10, 91054 Erlangen, Germany. ${ }^{2}$ Department of Pediatrics, Division of Neuropediatrics,

Friedrich-Alexander-Universität Erlangen-Nürnberg (FAU), Erlangen, Germany. ${ }^{3}$ Institute of Human Genetics, University of Regensburg, Regensburg, Germany.

Received: 17 October 2018 Accepted: 3 February 2019 Published online: 11 February 2019

\section{References}

1. Dutcher SK. Long-lost relatives reappear: identification of new members of the tubulin superfamily. Curr Opin Microbiol. 2003;6:634-40.

2. Hammond JW, Cai D, Verhey KJ. Tubulin modifications and their cellular functions. Curr Opin Cell Biol. 2008:20:71-6.

3. Kapitein LC, Hoogenraad CC. Building the neuronal microtubule cytoskeleton. Neuron. 2015;87:492-506.

4. Mandelkow E, Mandelkow EM. Microtubules and microtubule-associated proteins. Curr Opin Cell Biol. 1995:7:72-81.

5. Keays DA, et al. Mutations in alpha-tubulin cause abnormal neuronal migration in mice and lissencephaly in humans. Cell. 2007;128:45-57.

6. Abdollahi MR, et al. Mutation of the variant alpha-tubulin TUBA8 results in polymicrogyria with optic nerve hypoplasia. Am J Hum Genet. 2009:85:737-44.

7. Cushion TD, et al. De novo mutations in the beta-tubulin gene TUBB2A cause simplified gyral patterning and infantile-onset epilepsy. Am J Hum Genet. 2014:94:634-41.

8. Jaglin $\mathrm{XH}$, et al. Mutations in the beta-tubulin gene TUBB2B result in asymmetrical polymicrogyria. Nat Genet. 2009;41:746-52.

9. Poirier $\mathrm{K}$, et al. Mutations in the neuronal ss-tubulin subunit TUBB3 result in malformation of cortical development and neuronal migration defects. Hum Mol Genet. 2010;19:4462-73.

10. Breuss $M$, et al. Mutations in the beta-tubulin gene TUBB5 cause microcephaly with structural brain abnormalities. Cell Rep. 2012;2:1554-62.

11. Poirier $\mathrm{K}$, et al. Mutations in TUBG1, DYNC1H1, KIF5C and KIF2A cause malformations of cortical development and microcephaly. Nat Genet. 2013; 45:639-47.

12. Bahi-Buisson N, Cavallin M. Tubulinopathies overview. In: Adam MP, et al., editors. GeneReviews((R)). Seattle, Washington; 1993.

13. Bahi-Buisson N, et al. The wide spectrum of tubulinopathies: what are the key features for the diagnosis? Brain. 2014;137:1676-700.

14. Di Donato N, et al. Analysis of 17 genes detects mutations in $81 \%$ of 81 patients with lissencephaly. Genet Med. 2018;20:1354-64.
15. Morris-Rosendahl DJ, et al. Refining the phenotype of alpha-1a tubulin (TUBA1A) mutation in patients with classical lissencephaly. Clin Genet. 2008; 74:425-33.

16. Popp B, et al. Exome Pool-Seq in neurodevelopmental disorders. Eur J Hum Genet. 2017;25:1364-76.

17. Hauer NN, et al. Clinical relevance of systematic phenotyping and exome sequencing in patients with short stature. Genet Med. 2018;20:630-8.

18. Kircher $M$, et al. A general framework for estimating the relative pathogenicity of human genetic variants. Nat Genet. 2014;46:310-5.

19. Kohler S, et al. The human phenotype ontology in 2017. Nucleic Acids Res. 2017:45:D865-76.

20. Fallet-Bianco $C$, et al. Mutations in tubulin genes are frequent causes of various foetal malformations of cortical development including microlissencephaly. Acta Neuropathol Commun. 2014;2:69.

21. Landrum MJ, et al. ClinVar: improving access to variant interpretations and supporting evidence. Nucleic Acids Res. 2018;46:D1062-7.

22. Turner TN, et al. denovo-db: a compendium of human de novo variants. Nucleic Acids Res. 2017:45:D804-11.

23. Firth HV et al. DECIPHER: database of chromosomal imbalance and phenotype in humans using Ensembl resources. Am J Hum Genet. 2009;84: 524-33.

24. Wildeman M, van Ophuizen E, den Dunnen JT, Taschner PE. Improving sequence variant descriptions in mutation databases and literature using the Mutalyzer sequence variation nomenclature checker. Hum Mutat. 2008; 29:6-13.

25. Richards S, et al. Standards and guidelines for the interpretation of sequence variants: a joint consensus recommendation of the American College of Medical Genetics and Genomics and the Association for Molecular Pathology. Genet Med. 2015;17:405-24.

26. Li Q, Wang K. InterVar: clinical interpretation of genetic variants by the 2015 ACMG-AMP guidelines. Am J Hum Genet. 2017;100:267-80.

27. Hadley, W. ggplot2: Elegant graphics for data Analysis, (2016).

28. Vemu A, et al. Structure and dynamics of single-isoform recombinant neuronal human tubulin. J Biol Chem. 2016:291:12907-15.

29. Tischfield MA, Cederquist GY, Gupta ML Jr, Engle EC. Phenotypic spectrum of the tubulin-related disorders and functional implications of diseasecausing mutations. Curr Opin Genet Dev. 2011;21:286-94.

30. Kumar RA, et al. TUBA1A mutations cause wide spectrum lissencephaly (smooth brain) and suggest that multiple neuronal migration pathways converge on alpha tubulins. Hum Mol Genet. 2010;19:2817-27.

31. Zheng C, Diaz-Cuadros M, Nguyen KCQ, Hall DH, Chalfie M. Distinct effects of tubulin isotype mutations on neurite growth in Caenorhabditis elegans. Mol Biol Cell. 2017:28:2786-801.

32. Nogales E. Structural insight into microtubule function. Annu Rev Biophys Biomol Struct. 2001:30:397-420.

33. Li H, DeRosier DJ, Nicholson WV, Nogales E, Downing KH. Microtubule structure at 8 A resolution. Structure. 2002:10:1317-28.

34. Liu X, Jian X, Boerwinkle E. dbNSFP v2.0: a database of human nonsynonymous SNVs and their functional predictions and annotations. Hum Mutat. 2013;34:E2393-402.

35. Lek $\mathrm{M}$, et al. Analysis of protein-coding genetic variation in 60,706 humans. Nature. 2016;536:285-91.

36. Cingolani $P$, et al. A program for annotating and predicting the effects of single nucleotide polymorphisms, SnpEff: SNPs in the genome of Drosophila melanogaster strain w1118; iso-2; iso-3. Fly (Austin). 2012;6:80-92.

37. Team, R.D.C. R: A language and environment for statistical Computing. (2008).

38. Meyer, D., Zeileis, A., \& Hornik, K. vcd: Visualizing Categorical Data. (2017).

39. Iossifov I, et al. The contribution of de novo coding mutations to autism spectrum disorder. Nature. 2014;515:216-21.

40. Oegema R, et al. Recognizable cerebellar dysplasia associated with mutations in multiple tubulin genes. Hum Mol Genet. 2015;24:5313-25.

41. Poirier $\mathrm{K}$, et al. Large spectrum of lissencephaly and pachygyria phenotypes resulting from de novo missense mutations in tubulin alpha 1A (TUBA1A). Hum Mutat. 2007:28:1055-64.

42. Bahi-Buisson N, et al. Refinement of cortical dysgeneses spectrum associated with TUBA1A mutations. J Med Genet. 2008:45:647-53.

43. Jansen $A C$, et al. TUBA1A mutations: from isolated lissencephaly to familial polymicrogyria. Neurology. 2011;76:988-92.

44. Sohal AP, Montgomery T, Mitra D, Ramesh V. TUBA1A mutation-associated lissencephaly: case report and review of the literature. Pediatr Neurol. 2012; 46:127-31. 
45. Mokanszki A, et al. Lissencephaly and band heterotopia: LIS1, TUBA1A, and DCX mutations in Hungary. J Child Neurol. 2012;27:1534-40.

46. Cushion TD, et al. Overlapping cortical malformations and mutations in TUBB2B and TUBA1A. Brain. 2013;136:536-48.

47. Okumura A, et al. Lissencephaly with marked ventricular dilation, agenesis of corpus callosum, and cerebellar hypoplasia caused by TUBA1A mutation. Brain and Development. 2013;35:274-9.

48. Poirier $\mathrm{K}$, et al. Expanding the spectrum of TUBA1A-related cortical dysgenesis to Polymicrogyria. Eur J Hum Genet. 2013;21:381-5.

49. Zanni $\mathrm{G}$, et al. Description of a novel TUBA1A mutation in Arg-390 associated with asymmetrical polymicrogyria and mid-hindbrain dysgenesis. Eur J Paediatr Neurol. 2013;17:361-5.

50. Hikita $\mathrm{N}$, et al. A case of TUBA1A mutation presenting with lissencephaly and Hirschsprung disease. Brain and Development. 2014;36:159-62.

51. Romaniello R, et al. Brain malformations and mutations in alpha- and betatubulin genes: a review of the literature and description of two new cases. Dev Med Child Neurol. 2014;56:354-60.

52. Kamiya K, Tanaka F, Ikeno M, Okumura A, Aoki S. DTI tractography of lissencephaly caused by TUBA1A mutation. Neurol Sci. 2014;35:801-3.

53. Shimojima $\mathrm{K}$, et al. Whole-exome sequencing identifies a de novo TUBA1A mutation in a patient with sporadic malformations of cortical development: a case report. BMC Res Notes. 2014;7:465.

54. Yokoi $\mathrm{S}$, et al. TUBA1A mutation can cause a hydranencephaly-like severe form of cortical dysgenesis. Sci Rep. 2015;5:15165.

55. Myers KA, Bello-Espinosa LE, Kherani A, Wei XC, Innes AM. TUBA1A mutation associated with eye abnormalities in addition to brain malformation. Pediatr Neurol. 2015;53:442-4.

56. Bamba $Y$, et al. In vitro characterization of neurite extension using induced pluripotent stem cells derived from lissencephaly patients with TUBA1A missense mutations. Mol Brain. 2016;9:70.

57. Romaniello $R$, et al. Tubulin-related cerebellar dysplasia: definition of a distinct pattern of cerebellar malformation. Eur Radiol. 2017;27:5080-92.

58. Mencarelli A, et al. Epileptogenic brain malformations and mutations in tubulin genes: a case report and review of the literature. Int J Mol Sci. 2017;18:2273.

59. Fallet-Bianco C, et al. Neuropathological phenotype of a distinct form of lissencephaly associated with mutations in TUBA1A. Brain. 2008;131:2304-20.

60. Lecourtois M, et al. Human lissencephaly with cerebellar hypoplasia due to mutations in TUBA1A: expansion of the foetal neuropathological phenotype. Acta Neuropathol. 2010;119:779-89.

61. de Ligt J, et al. Diagnostic exome sequencing in persons with severe intellectual disability. N Engl J Med. 2012;367:1921-9.

62. Helbig KL, et al. Diagnostic exome sequencing provides a molecular diagnosis for a significant proportion of patients with epilepsy. Genet Med. 2016;18:898-905.

63. Lelieveld SH, et al. Meta-analysis of 2,104 trios provides support for 10 new genes for intellectual disability. Nat Neurosci. 2016;19:1194-6.

64. Posey JE, et al. Molecular diagnostic experience of whole-exome sequencing in adult patients. Genet Med. 2016;18:678-85.

65. Alby C, et al. Clinical, genetic and neuropathological findings in a series of 138 fetuses with a corpus callosum malformation. Birth Defects Res A Clin Mol Teratol. 2016;106:36-46.

66. Wiszniewski W, et al. Comprehensive genomic analysis of patients with disorders of cerebral cortical development. Eur J Hum Genet. 2018;26:1121-31.

67. Sato $T$, et al. A case of tubulinopathy presenting with porencephaly caused by a novel missense mutation in the TUBA1A gene. Brain and Development. 2018:40:819-23.

68. Nogales E, Wolf SG, Downing KH. Structure of the alpha beta tubulin dimer by electron crystallography. Nature. 1998;391:199-203.

69. Amrom D, et al. Polymicrogyria with dysmorphic basal ganglia? Think tubulin! Clin Genet. 2014;85:178-83.

70. Maday S, Twelvetrees AE, Moughamian AJ, Holzbaur EL. Axonal transport: cargo-specific mechanisms of motility and regulation. Neuron. 2014:84:292-309.

71. Kerjan G, Gleeson JG. Genetic mechanisms underlying abnormal neuronal migration in classical lissencephaly. Trends Genet. 2007;23:623-30.

72. Fokkema IF, et al. LOVD v.2.0: the next generation in gene variant databases. Hum Mutat. 2011;32:557-63.

73. Kikkawa M, Hirokawa N. High-resolution cryo-EM maps show the nucleotide binding pocket of KIF1A in open and closed conformations. EMBO J. 2006: 25:4187-94.

74. Kellogg EH, et al. Near-atomic model of microtubule-tau interactions. Science. 2018;360:1242-6.

Ready to submit your research? Choose BMC and benefit from:

- fast, convenient online submission

- thorough peer review by experienced researchers in your field

- rapid publication on acceptance

- support for research data, including large and complex data types

- gold Open Access which fosters wider collaboration and increased citations

- maximum visibility for your research: over $100 \mathrm{M}$ website views per year

At BMC, research is always in progress.

Learn more biomedcentral.com/submissions 